\title{
Influence of Row Spacing on Root Yield and Yield Components of Fodder Beet (Beta vulgaris var. crassa Mansf.) in the Black Sea Coastal Region
}

\author{
Sebahattin ALBAYRAK ${ }^{1}$ \\ Necdet ÇAMAŞ ${ }^{2}$
}

Geliş Tarihi: 22.11.2004

\begin{abstract}
Effects of four row spacing $(30,40,50$ and $60 \mathrm{~cm})$ on root yield and some yield components of fodder beet (Beta vulgaris var. crassa Mansf.) cultivar Ecdorot and cultivar Ecdogelb were evaluated in Çarşamba and Bafra plains, Turkey in the 2002 and 2003 growing seasons. The root yield, root dry matter rate, root dry matter yield, root diameter, root length, and sugar content were determined. Row spacing significantly affected most of the yield components determined in both locations. Root yield, root dry matter yield, root diameter, and root length increased along with increased of row spacing. However, root dry matter rate and sugar content were not greatly influenced by row spacing. The highest root dry matter was obtained from $50-60 \mathrm{~cm}$ row spacing for both cultivars and locations. Cultivar Ecdorat had higher yield data and this cultivar was also found more stable than cultivar Ecdogelb in the Black Sea costal region of Turkey.
\end{abstract}

Key Words: Fodder beet, row spacing, root yield, root dry matter yield

\section{Karadeniz Bölgesi Sahil Koşullarında Sıra Aralığının Yemlik Pancar (Beta vulgaris var. crassa Mansf.)'ın Yumru Verimi ve Verim Ögeleri Üzerine Etkisi}

Öz: 2002 ve 2003 yılları yetiştirme peryodunda, Çarşamba ve Bafra ovalarında dört farklı sıra aralığı $(30,40,50$ ve $60 \mathrm{~cm}$ )'nın Ecdorot ve Ecdogelb yemlik pancar çeşitlerinin yumru verimi ve bazı verim ögelerine etkileri incelenmiştir. Araştırmada, yumru verimi, yumru kuru madde oranı, yumru kuru madde verimi, yumru boyu, yumru çapı ve yumru şeker oranı incelenmiştir. Sıra aralığının artmasıyla yumru verimi, yumru kuru madde verimi, yumru boyu ve yumru çapı artmıştır. Buna karşıık yumru kuru madde oranı ve şeker içeriği sıra aralığından fazla etkilenmemiştir. Her iki lokasyonda ve çeşitte en yüksek kuru madde verimi 50-60 cm sıra aralığında bulunmuştur. Karadeniz Bölgesi sahil koşullarında, Ecdorot çeşidi Ecdogelb çeşidine göre daha stabil ve daha yüksek verim değerlerine sahip olduğu belirlenmiştir.

Anahtar Kelimeler: Yemlik pancar, sıra aralığı, yumru verimi, yumru kuru madde verimi

\section{Introduction}

Fodder beet is successively grown as a fodder crop in the coastal regions of many European countries. The plant is used as a valuable source of fodder for cattle (Niazi et al. 2000). Since fodder beet contains more water and sugar, it increases milk product and being suitable forage for dairy cows. The fodder beet is used by being chopped and by mixing with straw in European countries. It is also reported that the plant is suitable to make silage (Akyıldız 1983, Özen et al. 1993).

Including fodder beet in diet of cattle increases intake of dry matter that is quantitative and qualitative factors affecting intake of the basal diet. Vitamin and mineral supplements should be adjusted by increasing nitrogen supplements and decreasing energy from concentrate. Along with maize silage, $3 \mathrm{~kg}$ fodder beet with $5 \mathrm{~kg}$ hay per day is the optimum amount for cattle diet. It is also reported that milk contamination with butyric acid bacteria is lower when cows are fed with fodder beet compared to hay feed alone (Chenais 1994).

In the literature, various root yields of fodder beet are reported changing from 22.59 to $145.24 \mathrm{t} \mathrm{ha}^{-1}$ (Buryakov 1994, Rzekanowski 1994, Stroller 1994, Koszanski et al.
1995, JuSam et al. 1995, Podstawka and Ceglarek 1995, Drashkov 1996, Grzes et al. 1996, Lukic and Vasilijevic 1996, Avcıoğlu et al. 1999, Soya et al. 1999). The possible reasons of different root yields might be different ecological conditions, soil structure, light duration, varieties and cultural treatments. Common view in all these reports is that there is a significant correlation between row spacing and root yield. Specifically, the most suitable row spacing reported was 40-60 cm (Manga et al. 1997, Avcıoğlu et al. 1999, Soya et al. 1999, Acar and Mülayim 2001).

The aim of this study is to determine the effect of different row spacings of two fodder beet cultivars on root yield and its components in Çarşamba and Bafra plains located in Black Sea Coastal Region of Turkey.

\section{Materials and Methods}

Root yield and its components in fodder beet were examined in two different fodder beet cultivars at two locations in 2002 and 2003 in Turkey. Ecdorot and

\footnotetext{
${ }^{1}$ Karadeniz Tarımsal Araştırma Enstitüsü-Samsun

${ }^{2}$ Ondokuz Mayıs Üniv. Bafra Meslek Yüksekokulu-Samsun
} 
Ecdogelb are diploid fodder beet cultivars bred at Saaten Union, Germany in 1953. Fodder beet cultivars had production permission in 2002 in Turkey. Plants were grown under irrigated conditions in both locations. Sites are approximately $90 \mathrm{~km}$ apart. In the first experiment field located in Çarşamba, Samsun, the soil was clay loam, pH $7.1,952 \mathrm{~kg} \mathrm{ha}^{-1}$ potassium, $230 \mathrm{~kg} \mathrm{ha}^{-1}$ phosphorus, and middle organic matter $(2.42 \%)$.

The monthly rainfall for March through September was $34.1,61.9,10.9,53.8,79.9,14.3$ and $34.6 \mathrm{~mm}$ in 2002 ( $289.5 \mathrm{~mm}$ total) and 73.5, 45.0, 54.7, 3.3, 37.2, 3.4 and $94.0 \mathrm{~mm}$ in 2003 (311.1 $\mathrm{mm}$ total) for Çarşamba location. It was 24.9, 44.9, 6.2, 95.3, 6.2, 115.1 and 69.9 $\mathrm{mm}$ in 2002 (370.6 mm total) and 66.1, 40.5, 52.1, 9.5, 49.7, 3.2 and $135.2 \mathrm{~mm}$ in 2003 (356.3 mm total) for Bafra location. Factorial arrangements of four-row spacing (30, 40, 50 and $60 \mathrm{~cm}$ ) and two cultivars (Ecdorot and Ecdogelb) were evaluated in a randomized complete block design with three replications. Individual plot size was 2.4 $x 5 \mathrm{~m}=12 \mathrm{~m}^{2}$ except $50 \mathrm{~cm}$ which was $12.5 \mathrm{~m}^{2}$. Sowing was done by hand on 24 and 28 March in 2002 and 2003 in Çarşamba, 25 and 29 March in 2002 and 2003 in Bafra, respectively. As a fertilizer, calcium ammonium nitrate of $100 \mathrm{~kg} \mathrm{ha}^{-1}$ after sowing and $75 \mathrm{~kg} \mathrm{ha}^{-1}$ in May was uniformly applied to all plots as. There were no problems with pests, diseases or weeds during the course of study.

Ten plants from each replication were taken at harvest stage for morphological measurements. Root diameter and root length were measured from individual plants. The plots were harvested by hand after the roots matured in September and the other parameters were determined at the same time. All statistical analyses were conducted using GLM producers of SAS (1998). Means were compared using Least Significant Differences (LSD) tests at the 0.05 probability level. Mean yield of fodder beet cultivars $(x)$, regression coeffcient $(r)$, deviation from regression $\left(s^{2} d\right)$, determination coefficient $\left(r^{2}\right)$, coefficient variation $(\mathrm{CV})$, regression line intercept (a) were evaluated as stability parameters (Eberhart and Russell 1966).

\section{Results and Discussion}

The root yields of cultivars and row spacing were significantly different in both locations. The highest root yield $\left(115.46 \mathrm{t} \mathrm{ha}^{-1}\right)$ was obtained from Ecdorot with $50 \mathrm{~cm}$ row spacing in Çarşamba location, whereas the highest root yield $\left(110.41 \mathrm{t} \mathrm{ha}^{-1}\right)$ was recorded from Ecdorot with $60 \mathrm{~cm}$ row spacing in Bafra (Table 1 and Table 2). As it

Table 1. Effects of row spacing on root yield and its components of fodder beet in Çarşamba location

\begin{tabular}{|c|c|c|c|c|c|c|c|c|c|}
\hline \multirow{3}{*}{$\begin{array}{c}\text { Row } \\
\text { spacing } \\
\text { (cm) }\end{array}$} & \multicolumn{3}{|c|}{2002} & \multicolumn{3}{|c|}{2003} & \multicolumn{3}{|c|}{ average of 2 years } \\
\hline & ecdogelb & ecdorot & mean & ecdogelb & ecdorot & mean & ecdogelb & ecdorot & mean \\
\hline & \multicolumn{9}{|c|}{ Root yield (t ha $\left.{ }^{-1}\right)$} \\
\hline 30 & 73.17 & 83.67 & $78.42 \mathrm{c}$ & 85.95 & 90.30 & $88.12 \mathrm{~b}$ & 79.56 & 86.98 & $83.27 \mathrm{c}$ \\
\hline 40 & 80.48 & 96.11 & $88.30 \mathrm{~b}$ & 91.66 & 101.96 & $96.81 \mathrm{~b}$ & 86.07 & 99.04 & $92.55 \mathrm{~b}$ \\
\hline 50 & 101.0 & 112.07 & $106.53 \mathrm{a}$ & 109.74 & 118.85 & $114.29 \mathrm{a}$ & 105.37 & 115.46 & $110.41 \mathrm{a}$ \\
\hline 60 & 93.53 & 107.17 & $100.35 \mathrm{a}$ & 110.49 & 118.35 & $114.43 \mathrm{a}$ & 102.01 & 112.76 & $107.39 \mathrm{a}$ \\
\hline \multirow[t]{2}{*}{ mean } & $87.05 \mathrm{~b}$ & $99.75 \mathrm{a}$ & $93.40 \mathrm{~b}$ & $99.46 \mathrm{a}$ & $107.37 \mathrm{a}$ & $103.41 \mathrm{a}$ & $93.25 \mathrm{~b}$ & $103.56 \mathrm{a}$ & 98.41 \\
\hline & \multicolumn{9}{|c|}{ Root dry matter content (\%) } \\
\hline 30 & 13.52 & 12.26 & 12.89 & 11.55 & 12.45 & $12.00 \mathrm{~b}$ & 12.54 & 12.36 & 12.45 \\
\hline 40 & 11.86 & 12.72 & 12.29 & 11.59 & 12.99 & $12.29 \mathrm{ab}$ & 11.73 & 12.86 & 12.29 \\
\hline 50 & 12.58 & 13.61 & 13.09 & 12.57 & 12.84 & $12.70 \mathrm{a}$ & 12.58 & 13.23 & 12.90 \\
\hline 60 & 11.37 & 12.88 & 12.13 & 12.20 & 12.21 & $12.21 \mathrm{ab}$ & 11.79 & 12.55 & 12.17 \\
\hline \multirow[t]{2}{*}{ mean } & 12.33 & 12.87 & 12.60 & $11.98 \mathrm{~b}$ & $12.62 \mathrm{a}$ & 12.30 & 12.16 & 12.75 & 12.45 \\
\hline & \multicolumn{9}{|c|}{ Root dry matter yield (t ha $\left.{ }^{-1}\right)$} \\
\hline 30 & 9.93 & 10.19 & $10.06 \mathrm{c}$ & 9.93 & 11.41 & $10.67 \mathrm{~b}$ & 9.93 & 10.80 & $10.36 \mathrm{c}$ \\
\hline 40 & 9.46 & 12.19 & $10.82 \mathrm{bc}$ & 10.63 & 13.24 & $11.93 \mathrm{~b}$ & 10.04 & 12.71 & $11.38 \mathrm{c}$ \\
\hline 50 & 12.54 & 15.25 & $13.89 \mathrm{a}$ & 13.77 & 15.29 & $14.53 \mathrm{a}$ & 13.16 & 15.27 & $14.21 \mathrm{a}$ \\
\hline 60 & 10.59 & 13.72 & $12.16 \mathrm{~b}$ & 13.50 & 14.47 & $13.99 \mathrm{a}$ & 12.04 & 14.10 & $13.07 \mathrm{~b}$ \\
\hline \multirow[t]{2}{*}{ mean } & $10.63 \mathrm{~b}$ & $12.84 \mathrm{a}$ & $11.73 \mathrm{~b}$ & $11.96 \mathrm{~b}$ & $13.60 \mathrm{a}$ & $12.78 \mathrm{a}$ & $11.29 \mathrm{~b}$ & $13.22 \mathrm{a}$ & 12.26 \\
\hline & \multicolumn{9}{|c|}{ Root diameter $(\mathrm{cm})$} \\
\hline 30 & 8.28 & 8.66 & $8.47 \mathrm{c}$ & 10.34 & 9.46 & $9.89 \mathrm{c}$ & 9.31 & 9.06 & $9.18 \mathrm{~d}$ \\
\hline 40 & 9.17 & 9.28 & $9.22 \mathrm{bc}$ & 12.40 & 12.01 & $12.20 \mathrm{~b}$ & 10.79 & 10.64 & $10.71 \mathrm{c}$ \\
\hline 50 & 9.65 & 9.40 & $9.53 \mathrm{ab}$ & 13.88 & 14.62 & $14.25 \mathrm{a}$ & 11.77 & 12.01 & $11.89 \mathrm{~b}$ \\
\hline 60 & 9.96 & 10.29 & $10.12 \mathrm{a}$ & 14.39 & 15.69 & $15.04 \mathrm{a}$ & 12.17 & 12.99 & $12.58 \mathrm{a}$ \\
\hline \multirow[t]{2}{*}{ mean } & 9.27 & 9.40 & $9.33 \mathrm{~b}$ & 12.75 & 12.94 & $12.85 \mathrm{a}$ & 11.01 & 11.17 & 11.09 \\
\hline & \multicolumn{9}{|c|}{ Root length $(\mathrm{cm})$} \\
\hline 30 & 13.40 & 13.00 & $13.20 \mathrm{c}$ & 13.63 & 14.58 & $14.10 \mathrm{~d}$ & 13.51 & 13.79 & $13.63 \mathrm{c}$ \\
\hline 40 & 15.87 & 16.73 & $16.30 \mathrm{~b}$ & 17.39 & 18.50 & $17.94 \mathrm{c}$ & 16.63 & 17.62 & $17.12 \mathrm{~b}$ \\
\hline 50 & 18.13 & 17.33 & $17.73 \mathrm{ab}$ & 18.53 & 19.94 & $19.24 \mathrm{~b}$ & 18.33 & 18.64 & $18.48 \mathrm{a}$ \\
\hline 60 & 19.13 & 17.07 & $18.10 \mathrm{a}$ & 19.92 & 20.59 & $20.26 \mathrm{a}$ & 19.53 & 18.83 & $19.18 \mathrm{a}$ \\
\hline \multirow[t]{2}{*}{ mean } & 16.63 & 16.03 & $16.33 \mathrm{~b}$ & $17.37 \mathrm{~b}$ & $18.40 \mathrm{a}$ & $17.89 \mathrm{a}$ & 17.00 & 17.22 & 17.11 \\
\hline & \multicolumn{9}{|c|}{ Sugar content (\%) } \\
\hline 30 & 6.24 & 6.44 & 6.34 & 5.48 & 5.76 & 5.62 & 5.86 & 6.10 & $5.98 \mathrm{ab}$ \\
\hline 40 & 5.57 & 6.84 & 6.21 & 6.04 & 6.22 & 6.13 & 5.81 & 6.53 & $6.17 \mathrm{a}$ \\
\hline 50 & 5.88 & 6.12 & 5.99 & 5.42 & 5.86 & 5.64 & 5.65 & 5.99 & $5.82 a b$ \\
\hline 60 & 5.56 & 5.79 & 5.67 & 4.93 & 5.63 & 5.28 & 5.25 & 5.71 & $5.48 \mathrm{~b}$ \\
\hline mean & 5.81 & 6.30 & 6.05 & 5.47 & 5.87 & 5.67 & $5.64 \mathrm{~b}$ & $6.08 \mathrm{a}$ & 5.86 \\
\hline
\end{tabular}

Means followed by the same letter(s) and column(s) are not significantly different at the $p=0.05$ level. 
Table 2. Effects of row spacing on root yield and its components of fodder beet in Bafra location

\begin{tabular}{|c|c|c|c|c|c|c|c|c|c|}
\hline \multirow{3}{*}{$\begin{array}{c}\text { Row } \\
\text { spacing } \\
\text { (cm) }\end{array}$} & \multicolumn{3}{|c|}{2002} & \multicolumn{3}{|c|}{2003} & \multicolumn{3}{|c|}{ average of 2 years } \\
\hline & ecdogelb & ecdorot & mean & ecdogelb & ecdorot & mean & ecdogelb & ecdorot & mean \\
\hline & \multicolumn{9}{|c|}{ Root yield $\left(\mathrm{t} \mathrm{ha}^{-1}\right)$} \\
\hline 30 & 63.26 & 66.94 & $65.10 \mathrm{c}$ & 71.55 & 80.01 & $75.78 \mathrm{c}$ & 67.41 & 73.48 & $70.44 \mathrm{c}$ \\
\hline 40 & 75.22 & 79.29 & $77.26 \mathrm{~b}$ & 88.40 & 93.20 & $90.80 \mathrm{~b}$ & 81.81 & 86.24 & $84.03 \mathrm{~b}$ \\
\hline 50 & 92.03 & 102.15 & $97.09 \mathrm{a}$ & 103.71 & 114.11 & $108.91 \mathrm{a}$ & 97.87 & 108.13 & $103.0 \mathrm{a}$ \\
\hline 60 & 90.49 & 105.41 & $97.95 \mathrm{a}$ & 111.48 & 115.41 & $113.45 \mathrm{a}$ & 100.98 & 110.41 & $105.70 \mathrm{a}$ \\
\hline \multirow[t]{2}{*}{ mean } & $80.25 \mathrm{~b}$ & $88.45 a$ & $84.35 \mathrm{~b}$ & $93.79 \mathrm{~b}$ & $100.68 \mathrm{a}$ & $97.23 \mathrm{a}$ & $87.02 \mathrm{~b}$ & $94.57 \mathrm{a}$ & 90.79 \\
\hline & \multicolumn{9}{|c|}{ Root dry matter content (\%) } \\
\hline 30 & 10.59 & 11.19 & $10.89 \mathrm{~b}$ & 11.82 & 12.29 & 12.05 & 11.20 & 11.74 & $11.47 \mathrm{~b}$ \\
\hline 40 & 10.88 & 11.15 & $11.02 \mathrm{ab}$ & 12.64 & 13.99 & 12.23 & 11.76 & 12.57 & $12.17 \mathrm{ab}$ \\
\hline 50 & 10.79 & 12.45 & $11.62 \mathrm{a}$ & 11.68 & 14.36 & 13.02 & 11.24 & 13.41 & $12.32 \mathrm{a}$ \\
\hline 60 & 11.06 & 12.35 & $11.71 \mathrm{a}$ & 12.78 & 11.69 & 13.32 & 11.92 & 12.02 & $11.97 \mathrm{ab}$ \\
\hline \multirow[t]{2}{*}{ mean } & $10.83 \mathrm{~b}$ & $11.79 \mathrm{a}$ & $11.31 \mathrm{~b}$ & 12.23 & 13.08 & $12.66 \mathrm{a}$ & $11.53 \mathrm{~b}$ & $12.43 \mathrm{a}$ & 11.98 \\
\hline & \multicolumn{9}{|c|}{ Root dry matter yield $\left(\mathrm{t} \mathrm{ha}^{-1}\right)$} \\
\hline 30 & 6.69 & 7.49 & $7.09 \mathrm{c}$ & 8.45 & 9.79 & $9.12 \mathrm{c}$ & 7.57 & 8.64 & $8.11 \mathrm{c}$ \\
\hline 40 & 8.18 & 8.85 & $8.52 \mathrm{~b}$ & 11.19 & 13.07 & $12.13 \mathrm{~b}$ & 9.68 & 10.96 & $10.32 \mathrm{~b}$ \\
\hline 50 & 9.93 & 12.72 & $11.32 \mathrm{a}$ & 12.09 & 16.34 & $14.22 \mathrm{a}$ & 11.01 & 14.53 & $12.77 \mathrm{a}$ \\
\hline 60 & 10.01 & 13.01 & $11.51 \mathrm{a}$ & 14.23 & 13.49 & $13.86 \mathrm{ab}$ & 12.12 & 13.25 & $12.69 \mathrm{a}$ \\
\hline \multirow[t]{2}{*}{ mean } & $8.70 \mathrm{~b}$ & $10.52 \mathrm{a}$ & $9.61 \mathrm{~b}$ & $11.49 \mathrm{~b}$ & $13.17 \mathrm{a}$ & $12.33 \mathrm{a}$ & $10.10 \mathrm{~b}$ & $11.85 \mathrm{a}$ & 10.97 \\
\hline & \multicolumn{9}{|c|}{ Root diameter (cm) } \\
\hline 30 & 8.97 & 9.24 & $9.10 \mathrm{~b}$ & 9.65 & 9.12 & $9.39 \mathrm{~b}$ & 9.31 & 9.18 & $9.25 \mathrm{~b}$ \\
\hline 40 & 8.50 & 10.74 & $9.62 \mathrm{~b}$ & 9.85 & 10.16 & $10.01 \mathrm{~b}$ & 9.17 & 10.45 & $9.81 \mathrm{~b}$ \\
\hline 50 & 10.11 & 11.30 & $10.71 \mathrm{ab}$ & 11.73 & 12.09 & $11.91 \mathrm{a}$ & 10.92 & 11.70 & $11.31 \mathrm{a}$ \\
\hline 60 & 12.27 & 12.08 & $12.18 \mathrm{a}$ & 12.05 & 13.08 & $12.57 \mathrm{a}$ & 12.16 & 12.58 & $12.37 \mathrm{a}$ \\
\hline \multirow[t]{2}{*}{ mean } & 9.96 & 10.84 & 10.40 & 10.83 & 11.11 & 10.97 & 10.39 & 10.98 & 10.68 \\
\hline & \multicolumn{9}{|c|}{ Root length (cm) } \\
\hline 30 & 13.57 & 14.49 & $14.03 \mathrm{~b}$ & 14.26 & 13.86 & $14.06 \mathrm{~b}$ & 13.91 & 14.17 & $14.04 \mathrm{~b}$ \\
\hline 40 & 15.67 & 17.44 & $16.56 \mathrm{ab}$ & 15.79 & 17.21 & $16.50 \mathrm{a}$ & 15.73 & 17.33 & $16.53 \mathrm{a}$ \\
\hline 50 & 17.42 & 17.35 & $17.39 \mathrm{a}$ & 16.93 & 17.99 & $17.46 \mathrm{a}$ & 17.18 & 17.67 & $17.42 \mathrm{a}$ \\
\hline 60 & 18.99 & 17.26 & $18.13 \mathrm{a}$ & 18.71 & 17.82 & $18.26 \mathrm{a}$ & 18.85 & 17.54 & $18.20 \mathrm{a}$ \\
\hline \multirow[t]{2}{*}{ mean } & 16.41 & 16.64 & 16.53 & 16.42 & 16.72 & 16.57 & 16.42 & 16.68 & 16.55 \\
\hline & \multicolumn{9}{|c|}{ Sugar content (\%) } \\
\hline 30 & 5.24 & 5.84 & 5.54 & 5.13 & 6.43 & 5.78 & 5.19 & 6.14 & 5.66 \\
\hline 40 & 5.37 & 5.54 & 5.46 & 5.71 & 7.15 & 6.43 & 5.54 & 6.35 & 5.94 \\
\hline 50 & 5.38 & 5.72 & 5.55 & 6.17 & 6.85 & 6.51 & 5.78 & 6.29 & 6.03 \\
\hline 60 & 5.16 & 5.70 & 5.43 & 5.64 & 6.18 & 5.91 & 5.40 & 5.94 & 5.67 \\
\hline mean & $5.29 \mathrm{~b}$ & $5.70 \mathrm{a}$ & $5.49 \mathrm{~b}$ & $5.66 \mathrm{~b}$ & $6.65 \mathrm{a}$ & $6.16 \mathrm{a}$ & $5.48 \mathrm{~b}$ & $6.18 \mathrm{a}$ & 5.83 \\
\hline
\end{tabular}

Means followed by the same letter(s) and column(s) are not significantly different at the $p=0.05$ level.

was indicated before, the root yield range from 22.59 to $145.24 \mathrm{t} \mathrm{ha}^{-1}$ in fodder beet (Buryakov 1994, Rzekanowski 1994, Stroller, 1994, Koszanski et al. 1995, JuSam et al 1995, Podstawka and Ceglarek 1995, Drashkov 1996, Grzes et al. 1996, Lukic and Vasilijevic 1996, Avcıoğlu et al. 1999, Soya et al. 1999). Fodder beet when grown under suitable conditions, can produce $150-200 \mathrm{t} \mathrm{ha}^{-1}$ root yield (Açıkgöz 1991, Adıyaman 1996).

As row spacing widens, life area of plants increases. So using of nutrition matter, including water, light etc. per plant also increases. Increased photosynthetic activity leads to increase in root yield by increasing root diameter, root length, and carbohydrate storage (Salisbury and Rose 1992, Avcıoğlu 1995). The lowest average dry matters of roots were $12.17 \%$ with $60 \mathrm{~cm}$ and $11.47 \%$ with $30 \mathrm{~cm}$ row spacing in Çarşamba and Bafra, respectively. Previous results indicated that there was a negative correlation between root yield and rate of root dry matter in fodder beet (Langer and Hill 1991, Adıyaman 1996, Geren and Avcıoğlu 1996, Öz and Avcıoğlu 1997, Soya et al. 1997). It is also stated that there might be slight changes in root dry matter contents, as row spacing widens (Avcıoğlu et al. 1999, Soya et al. 1999). Although there were no statistically significant differences among the rates of root dry matter, it was relatively lower in wide row spacing than narrow row spacing. Ecdorot gave a higher dry matter rate than Ecdogelb in both locations (Table 1 and Table 2). It was previously reported that rates of dry matter in fodder beet might change from $11.82 \%$ to 18.60 $\%$ (Rzekonowski 1994, Lukic and Vasilijevic 1996, Avcıoğlu et al. 1999, Soya et al. 1999).

The highest dry matter yield was obtained from cultivar Ecdorot (15.25 and $15.29 \mathrm{t} \mathrm{ha}^{-1}$, respectively) in both years in $50 \mathrm{~cm}$ row spacing at Çarşamba location. At Bafra location, in the first year, while the highest root dry matter yield was obtained from cultivar Ecdorot $(13.01 \mathrm{t}$ $\mathrm{ha}^{-1}$ ) in $60 \mathrm{~cm}$ row spacing. In the second year, cultivar Ecdorot had the highest yield $\left(16.34 \mathrm{t} \mathrm{ha}^{-1}\right)$ in $50 \mathrm{~cm}$ row spacing (Table 1 and Table 2).

The root dry matter yields in the second year were higher than yields of the first year in both locations. These differences might be caused by the ecological conditions, such as precipitation and temperature recorded during the vegetative growth cycle. Avcıoğlu et al. (1999) showed that the variation seen in dry matter rates of fodder beet is 
also one of the reasons for the variation seen in root dry matter yield. In addition, several researches indicated that in contrast to root yield increase, both dry matter rate and root dry matter yield are decreased with a wide row space application (Geren and Avcıoğlu 1996, Öz and Avcıoğlu 1997, Soya et al. 1999, Acar and Mülayim 2001). Avcıoğlu et al. (1999) reported that increasing row spacing increased dry matter yield. Our results are similar to Avcıoğlu et al. (1999).

As row spacing widen, root diameter is also increased in cultivars Ecdorot and Ecdogelb in both locations (Table 1 and Table 2). Moreover, as the plant area widens, plant diameter is also getting increase. Since high plant density limits nutrition uptake and photosynthesis activity, plants are not able to store enough nutrition to root due to competition. Therefore, root development is restricted (Avcıoğlu et al. 1999; Soya et al. 1999; Geren and Avcıoğlu 1996; Sağlamtimur and Tansı, 1989). On the other hand, Sağlamtimur et al. (1989) reported a negative correlation between root diameter and plant density.

As row spacing is widening, root length is also increased in both cultivars in both locations, just like the root diameter (Table 1 and Table 2). Avcıoğlu et al. (1999) and Soya et al. (1999) indicated that root length was increased as the row spacing widen.

The lowest average sugar content was obtained from 60 and $30 \mathrm{~cm}$ row spacing in Çarşamba (5.48 \%) and
Bafra locations (5.66\%), respectively. Tayşi and Demir, (1979) reported that there was a negative correlation between sugar content and root yield, whereas root dry matter rate is positively correlated with sugar content.

If genotypes' regression coeffcient $(r)$ is close to 1 , regression line intercept $(a)$ is possitive, determination coefficient $\left(r^{2}\right)$ is close to 1 , deviation from regression $\left(s^{2} d\right)$ is close to 0 , coefficient variation (CV) is low, genotypes are stable in all this conditions (Eberhart and Russell 1966). According to this literature, cultivar Ecdorot is more stable than cultivar Ecdogelb (Table 3 and Figure 1).

According to the research investigated different row spacing on root yield and yield components of fodder beet (Beta vulgaris var. crassa Mansf.) cultivars having production permission in 2002 in Turkey in Çarşamba and Bafra plains located Blacksea Coastal Region in Turkey, the highest root dry matter was obtained from $50-60 \mathrm{~cm}$ row spacing for both cultivars and locations. Cultivar Ecdorat had higher yield data and this cultivar was found more stable than cultivar Ecdogelb too.

Table 3. Stability parameters for root dry matter yields

\begin{tabular}{|l|c|c|c|c|c|c|}
\hline Cultivars & $\mathbf{x}$ & $\mathbf{b}$ & $\mathbf{a}$ & $\mathbf{r}^{2}$ & $\mathbf{C V}$ & $\mathbf{S}^{\mathbf{2}} \mathbf{d}$ \\
\hline Ecdogelb & 10.69 & 1.03 & -1.25 & 0.996 & 0.66 & 0.005 \\
\hline Ecdorot & 12.53 & 0.93 & 1.739 & 0.991 & 0.78 & 0.01 \\
\hline
\end{tabular}

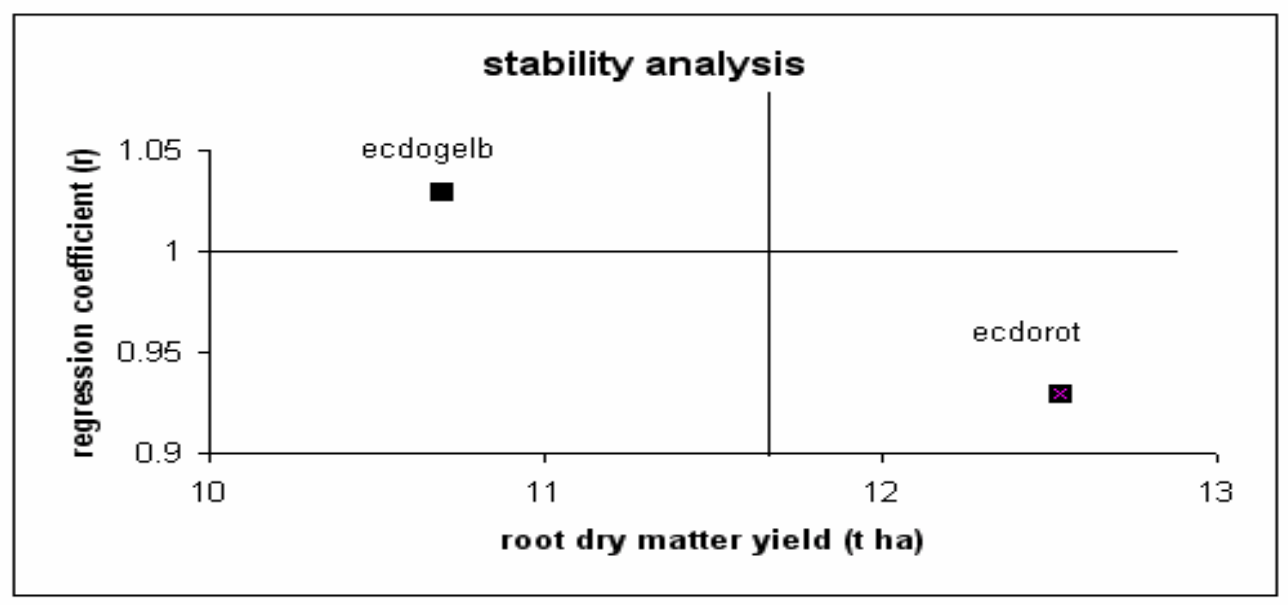

Figure 1. Stability situation of fodder beet cultivars according to root dry matter yield and regression coefficient

\section{References}

Acar, R. ve M. Mülayim. 2001. Hayvan pancarı (Beta vulgaris var. rapacea Koch.) çeșitlerinde farklı ekim zamanı ve bitki sıklığının kuru madde verimi ve bazı verim komponentleri üzerine etkileri. Türkiye 4. Tarla Bitkileri Kongresi. 85-90.

Açıkgöz, E. 1991. Yembitkileri. Uludağ Univ. Ziraat Fakültesi. No: 7-025-0210, Bursa. 456s.
Adıyaman, M. 1996. Hayvan Pancarı. Pan tohum geliştirme ve üretim şirketi. Mithatpaşa caddesi. No: 19/2. PK.19, Yenişehir, Ankara.

Akyıldız, A. R. 1983. Yemler Bilgisi ve Teknolojisi. Ankara Üniv . Ziraat Fak. No: 868. Ankara. 
Avcıoğlu, R., H. Geren, Y. Elmalı ve O. Erekul. 1999. Hayvan pancarı'ında (Beta vulgaris var. rapacea Koch.) farklı ekim zamanı ve bitki yoğunluğunun verim ve bazı verim öğeleri üzerine etkilerinin belirlenmesi üzerine araştırmalar. Türkiye 3. Tarla Bitkileri Kongresi. 113-118.

Avcıoğlu, R. 1995. Bitki Fizyolojisi. Ders notları. Ege Univ. Ziraat Fak. 200s. İzmir.

Buryakov, A. T. 1994. A screw-type cleaner for fodder beet. Zemledelie. No 3, 27. Russia.

Cenais, V. 1994. Use of fodder beet in the feeding of dairy cows. Bulletin des G.T.V. No 2: 49-56. France.

Drashkov, N. 1996. Results of purfied sofia waste waters in fodder beet irrigation. Pochvoznanie, Agrokhimiia y ekologiya. 31 (3): 36-39, Bulgaria.

Eberhart, S. A and W. A. Russell. 1966. Stability parameters for comparing varieties. Crop Sci. 6: 36-40.

Geren, H. and R. Avcıoğlu. 1996. Farklı hayvan pancarı (Beta vulgaris var. rapacea Koch.) çeşitlerinde farklı ekim zamanlarının verim ve verim komponentleri üzerine etkileri. Ege Univ. Yüksek lisans Tezi. 70s.

Grzes, S., S. Sobiech, T. Maciejewski and J. Szukula. 1996. Yield od fodder beet as influenced by sprinkler irrigation, method of sowing the preceeding crop and nitrogen fertilizer application. Prace Zakresu Nauk Rolniczych. 81: 87-94. Poland.

JuSam, L., A. JongHo, J. IkHwan, R. ZinSik, S. ByoungDon, P. MooKyun, J. H. Ahn, I. H. Jo, Z. S. Rho, B. D. Sang and M. K. Park. 1995. Effect of sowing date on the fresh yield of fodder beet cultivars. Journal of the Korean Society of Grassland Science. 15 (2): 140-145.

Koszanski, Z., S. Karczmarczyk and M. Roy. 1995. Effect of sprinkler irrigation and nitrogen fertilizer application on sugar an fodder beets cultivated on a good rye complex soil. I. Yields. Zeszyty Naukowe Akademii Rolniczej w Szczecinie, Rolnictwo. 59: 57-64. Poland.

Langer, R. H. and G. H. Hill. 1991. Agricultural plants. Plant Science Department, Lincoln Univ, New Zealand, Cambridge Univ. Press.

Lukic, D. and S. Vasilijevic. 1996. Production characteristics of some domestic and foregin fodder beet cultivars. Selekcija $i$ Semenarstvo. 3 (3-4): 86-91 Yugoslavia.

Manga, İ., Z. Acar, İ. Ayan, İ. Tiryaki and M. A. Özyazıcı. 1997. Samsun sulu ve susuz košllarında hayvan pancarı (Beta vulgaris var. rapacea Koch.)'inda mineral ve hayvan gübresi, ekim zamanı ve bitki yoğunluğunun verim ve baz kalite özelliklerine etkileri. Türkiye 2. Tarla Bitkileri Kongresi. 482-486.

Niazi, B. H., J. Rozema, R. A. Broekman and M. Salim. 2000 Dynamics of growth and water relations of fodder beet and sea beet in response to salinity. J. Agronomy and Crop Science 184: 101-109.
Öz, F. and R. Avcıoğlu. 1997. Hayvan pancarı (Beta vulgaris var. rapacea Koch.)'ında ekim oranı ve farklı yükseltilerin verim ve bazı verim ögeleri üzerine etkileri. Ege Univ. Yüksek Lisans Tezi.

Özen, N., A. Çakır, S. Haşimoğlu ve S. Aksoy. 1993. Yemler Bilgisi ve Yem Teknolojisi Ders Notları: 50. Atatürk Univ . Erzurum.

Podstawka, E. and S. Ceglarek. 1995. Cultivation of sugarbeet and fodder beet on light soil under conditions of sprinkler irrigation and differentiated nitrogen fertilizer application. Annales Universitatis Mariae Curie Sklodowska. Sectio E, Agricultura 50: 21-27. Poland.

Rzekanowski, C. 1994. The reaction of fodder beet grown on very ligth soil so sprinkler irrigation and nitrogen fertilizer application. Roczniki Nauk Rolniczych. Seria F, melioracji i Uzytkow Zielonych. 83 (3-4): 57-62. Poland.

Sağlamtimur, T. ve V. Tansı. 1989. Çukurova'da hayvan pancarında en uygun ekim zamanının belirlenmesi üzerine bir araştırma. Çukurova Üniv. Ziraat Fak. Dergisi. 4 (1): 6275 .

Sağlamtimur, T., V. Tansı ve H. Baytekin. 1989. Yembitkileri Tarımı. Çukurova Unıv. Ziraat Fak. Ders notları. 219-221.

Salisbury, F. B. and C. W. Rose. 1992. Plant Physiology, Wadsworth Pub. Com., Inc., Belmont, California-USA.

SAS, SAS/STAT 1998. User's Guide.Version 7.0. SAS Institute, Inc.Cary. NC.USA.

Soya, H., R. Avcıoğlu, H. Geren, F. Öz ve A. Öz. 1999. Hayvan pancarı (Beta vulgaris var. rapacea Koch.)'ında farklı yüksekliklerde ekim zamanı ve ekim oranının verim ve verim komponentleri üzerine etkileri. Türkiye 2. Tarla Bitkileri Kongresi. 284-288.

Soya, H., R. Avcıoğlu ve H. Geren, 1997. Yembitkileri. Hasat Yayınları. P.K. 212. Kadıköy-İstanbul.

Stroller, J. 1994. Stand structure and yields of fodder beet grown without manual work. Sbornik Vysoke Zemedelske v Praze, Fakulta Agronomicka, Rada A, Rostlinna Vyroba. 56: 99102. Czech Republic.

Tayşi, V. ve I. Demir, 1979. Diploid ve Polyploid Hayvan Pancarının Islahı. Ege Üniv. Ziraat Fak. Yayınları. 51s.

İletişim adresi:

Sebahattin ALBAYRAK

Karadeniz Tarımsal Araştırma Enstitüsü-Samsun

Tel: 03622560514

e-posta: sebo_albayrak@yahoo.com 